EPJ Web of Conferences 23, 00018 (2012)

DOI: $10.1051 /$ epjconf/20122300018

(c) Owned by the authors, published by EDP Sciences, 2012

\title{
Transport measurements on ultra-clean dual-gated suspended bilayer graphene
}

\author{
J. Velasco Jr. ${ }^{1}$, L. Jing ${ }^{1}$, Y. Lee ${ }^{1}$, P. Kratz ${ }^{2}$, W. Bao ${ }^{1}$, D. Smirnov ${ }^{3}$, M. Bockrath ${ }^{1}$ and C. N. Lau \\ ${ }^{1}$ Department of Physics and Astronomy, University of California, Riverside, CA 92521, USA \\ ${ }^{2}$ Department of Physics and Astronomy, Stanford University, Stanford, CA 94305, USA \\ ${ }^{3}$ National High Magnetic Field Laboratory, Tallahassee, FL 32310, USA
}

\begin{abstract}
We fabricate ultra clean contactless dual-gated devices comprised of suspended bilayer graphene (BLG) and investigate their low temperature electron transport properties as functions of charge density $n$, magnetic field $B$ and electric field $E_{\perp}$. We find that for devices with moderate mobility, conductance remains finite at the charge neutrality point and decreases monotonically with increasing $\left|E_{\perp}\right|$, as expected in the picture of single particle behavior. In contrast, devices with highest mobility display an insulating state at $B=E_{\perp}=n=0$, and conductance's dependence on $E_{\perp}$ is non-monotonic. This surprising behavior arises from electronic interactions. In finite $B$, we observe quantum Hall plateaus with the 8-fold degeneracy of the lowest Landau level completely broken, as well as a fractional state.
\end{abstract}

\section{Introduction}

The novel advancement of isolating graphene [1,2] single sheets of carbon, by mechanical exfoliation onto insulating substrates has produced an unabated flurry of scientific investigation that continue unabated to today. This "wonder material" has garnered such attention due to its capability for both real world applications and fundamental scientific exploration. Graphene's fantastic material properties, such as high mobility, high thermal conductivity, pristine crystallographic quality, and atomically thin and flexible conducting membrane, poise it as a replacement of silicon for graphene based electronics such as transistors, sensors, displays and photovoltaics. Scientifically, graphene has a linear dispersion relation, and has emerged as a table-top platform for relativistic physics [3, 4], electron optics [5] and many-body phenomena [6-8].

Single layer graphene (SLG), however, is not without drawbacks. It lacks a band gap that is critical for digital electronic applications, and electronic interactions are relatively weak [9]. Bilayer graphene (BLG) [10,11] two Bernal-stacked sheets of SLG, has become increasingly important. It has a quadratic dispersion relation with valence and conduction bands that touch at a point. As a result, its charge carriers are massive Dirac particles, and it has a band gap that can be tuned from 0 to $250 \mathrm{meV}$ by a perpendicular electric field [11,12]. Furthermore, recent advances in sample fabrication have allowed access to many-body physics [13-16], leading to, within the past year, reports of broken symmetry states [17], fractional quantum Hall effect (FQHE) [18], an intrinsic gap at the Dirac point [19] and spectrum reconstruction [20]. The latter two phenomena are attributed to the competition between symmetries for the ground state BLG. Therefore, BLG provides a fantastic platform for both real world electronics and exploration of new phases of matter.
In this report we will discuss our transport studies of ultra-clean, suspended BLG with double gates. We will start by describing device fabrication, followed by discussion of device annealing for achieving high mobility. Transport data will then be presented. By comparing results from devices with different mobility and intrinsic doping, we demonstrate the distinct signatures of single particle physics and an interactioninduced correlated phase. Lastly, we explore the devices' quantum Hall effect in magnetic fields.

\section{Device Fabrication}

Bilayer graphene (BLG) sheets are exfoliated onto $\mathrm{Si}_{2} \mathrm{O}_{2}$ wafers and identified using optical contrast. Suspended top gates are first fabricated across selected BLG sheets, using a multi-level lithography technique that was described in detail in [21, 22]. The key differences from the prior works are: (1) Instead of titanium, chromium is used to ensure the suspended top gate survives the etching treatment; (2) The height of the top gate is designed to be $\sim 300 \mathrm{~nm}$ and the thicknesses of the 3 angled depositions are 150, 200 and $100 \mathrm{~nm}$, respectively, to ensure structural integrity. After the top gate fabrication, the graphene sheets are coupled to electrodes, which consist of $10 \mathrm{~nm}$ of $\mathrm{Cr}$ and $120 \mathrm{~nm}$ of $\mathrm{Au}$, by standard electron beam lithography. The completed devices are then immersed in a buffered oxide etch (BOE 6:1) for 70 seconds, so as to release the BLG sheets from the $\mathrm{SiO}_{2}$ substrates, and dried in a critical point dryer. A typical device is $1.3 \mathrm{~mm}$ long and 1-1.3 $\mathrm{mm}$ wide. Successful devices are measured in our He3 cyrostat using a standard lock-in technique. 


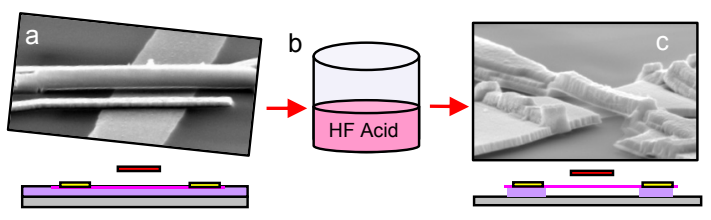

Fig. 1. SEM images and schematics of device fabrication. a. Graphene (pink) resting on $\mathrm{SiO}_{2}$ substrate (lavender) is coupled with $\mathrm{Cr} / \mathrm{Au}$ electrodes(yellow), while chrome contactless top gate (red) straddles graphene flake. b. Red arrow illustrates completed device in (a) is immersed in hydrofluoric acid and then dried with critical point dryer (not shown). c. Using the same

\section{Device Characterization and Mobility}

As-fabricated suspended graphene samples require current annealing to improve their quality [23-25]. This is performed in our cyrostat under vacuum at $T=\sim 1 \mathrm{~K}$. Usually the device is annealed in several stages, with the final stage determined by device mobility, minimum conductivity value, and proximity of the Dirac point to zero gate voltage. Details of this procedure can be found in [19].

The result of a successfully annealed device is shown in figures 2a-b, where we plot the device resistivity $r$ as a function of back gate voltage $V_{b g}$ and top gate voltage $V_{t g}$, respectively. The device has very low resistivity at large negative (positive) gate voltages, corresponding to hole (electron) doped regimes. Close to zero gate voltage, each of the curves display a dramatic peak, corresponding to the Dirac point or charge neutrality point (CNP) of the device, at which the device is nominally undoped. The gate voltages are related to induced charge density $n$ by

$n_{b g}=\alpha\left(V_{b g}-V_{b g}^{\prime}\right)$

$n_{t g}=b\left(V_{t g}-V_{t g}^{\prime}\right)$

where $a$ and $b$ are the gate coupling efficiencies for back and top gates, respectively, and $V_{t g}^{\prime}$ and $V_{b g}^{\prime}$ are the gate voltages values at which CNP is reached. For the device shown here, both $V_{t g}^{\prime}$ and $V_{b g}^{\prime}<0.01 \mathrm{~V}$. From Landau fan diagrams (see discussion below) as well as from geometrical considerations, we estimate $a \sim 1.83 \times 10^{10}$ and $b \sim 3.14 \times 10^{10} \mathrm{~cm}^{-2} \mathrm{~V}^{-1}$.

The high quality of the device can be discerned from two figures of merit. (1) Its field effect mobility, $\mu=\frac{1}{e} \frac{d \sigma}{d n}$, reaches $60,000 \mathrm{~cm}^{2} / \mathrm{Vs}$, suggesting quasiballistic transport of the charge carriers in the BLG from source to drain electrode. Here $e$ is the electron charge and $\sigma=1 / \rho$ is the conductivity. Moreover, (2) the CNP of the device displays clear electron-hole symmetric $r\left(V_{g}\right)$ curves, suggesting minimal doping from charge impurities [26, 27]. For our devices, both mobility and CNP voltages are comparable to those reported in prior studies of suspended BLG [18, 28], and are exceptional for devices with dual gated geometry [17].

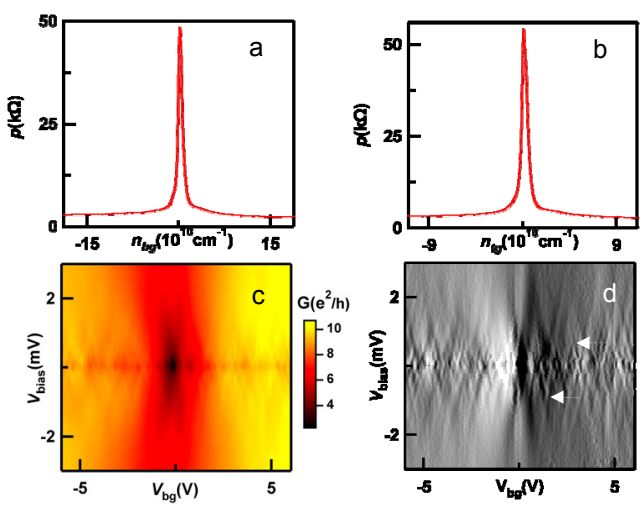

Fig. 2. Single gate transport data at $\mathrm{B}=0$ and $\mathrm{T}=300 \mathrm{mK}$ a. Resistivity plotted as a function of charge carrier density that is modulated by back gate. b. Resistivity plotted as a function of charge carrier density that is modulated by top gate. c. Differential conductance(color scale) plotted as a function of back gate voltage and source-drain voltage bias. d. Differential conductance differentiated with respect to $\mathrm{V}_{\mathrm{bg}},\left(\mathrm{dG}_{\mathrm{d}} \mathrm{dV} \mathrm{bg}\right)$, and plotted as a function of back gate voltage and source-drain voltage bias.

\section{Fabry-Perot Oscillations}

We first study the transport properties of the device in zero magnetic field. To acquire insight into the quasiballistic charge transport of the sample, we measured the differential conductance $G$ as a function of $V_{b g}$ and source drain bias $V$. The result is displayed in figure $2 \mathrm{~d}$, which exhibits several striking features. In the region of $V=V_{b g}$ $=0$, a feature resembling a dark $\mathrm{X}$ is seen, corresponding to the Dirac point, where the resistance reaches $50 \mathrm{k} \Omega$ and as high as $1 \mathrm{M} \Omega$ in some devices. Such large resistance corresponds to a symmetry-broken insulating state in charge neutral graphene, and is studied in detail in [19]. In regions of nonzero $V_{b g}$ and small finite $V$ additional features emerge. In order to accentuate such features, we plot $d G / d V_{b g}$, conductance differentiated with respect to $V_{b g}$, as functions of $V$ and $V_{b g}$ (figure 2e). Here we can clearly see criss-crossing lines forming a series of quasi-periodic, nesting diamonds, indicating the conductance oscillates with respect to both gate voltage and source drain bias.

Such observations can be explained as a manifestation of the quantum interference of multiply- reflected trajectories of electrons and hole waves between two partially transmitting electrodes. By tuning either $V$ or $V_{b g}$, the charges' wavelengths are altered, thus modulating the interference pattern. Therefore, the suspended BLG acts a Fabry-Perot resonant cavity for the charge carrier waves. Though similar observations were reported for substrate supported single layer graphene $[29,30]$, and carbon nanotubes [31], it has not been observed in suspended BLG before.

The period of the Fabry-Perot conductance oscillation is determined by the condition that the charges accumulate a phase of $2 p$ after completing a round-trip within the cavity. BLG's dispersion relation is 
$E=\frac{\hbar^{2} k^{2}}{2 m^{*}}$

where $\hbar$ is the Planck constant, $k$ is the wave vector, $m^{*} \sim 0.03 m_{e}$ is the effective mass, and $m_{e}$ is the rest mass of an electron. Thus, the conductance oscillation will have a characteristic energy

$E_{c}=\frac{\left(\frac{h}{2 L}\right)^{2}}{2 m^{*}}$,

where $L$ is the length of the cavity. From the typical size $V_{c}$ of the diamonds, which is indicated by the white arrow, we can extract a characteristic energy scale $E_{c}=$ $e V_{c} \sim 0.5-1 \mathrm{meV}$. This suggests that the electrons' phase coherence length is $>\sim 700 \mathrm{~nm}$, since the charges must maintain phase coherence for at least a distance of $2 L$ to exhibit Fabry-Perot oscillation. This demonstrates that the charges are phase coherent through the length of the device. The nested self-similar structures of the diamonds are a signature of the two dimensional nature of the cavity, since many different modes can be excited, resulting in the observation of several characteristic energy scales.

\section{Transport Properties in the Presence of Electric Field}

Sweeping both top and back gate voltages allows for independent modulation of the electric field $E_{\perp}$ and total charge carrier density $n$ in BLG. These 2 parameters can be calculated from $V_{b g}$ and $V_{t g}$ using the following relations,

$$
\begin{aligned}
& E_{\perp}=\left(n_{b g}-n_{t g}\right) \frac{e}{2 \varepsilon_{0}} \\
& n=n_{b g}+n_{t g}
\end{aligned}
$$

where $e_{o}$ is the permittivity of vacuum, and $n_{b g}$ and $n_{t g}$ are calculated from Eq. (1).

We now focus on the behaviors of two different devices. Sample 1 has mobility $20,000 \mathrm{~cm}^{2} / \mathrm{Vs}$, and $V_{b g}=-0.74 \mathrm{~V}$. Figure $2 \mathrm{a}$ plots $G$ from sample 1 as a function of $E_{\perp}$ and $n$. The red vertical band corresponds to the local conductance minimum at $n=0$ for a given $E_{\perp}$. Line traces $G(n)$ at different $E_{\perp}$ values are shown in Fig. 2b. We can see that $G \sim 150 \mu \mathrm{S}$ at $n=E_{\perp}=0$, but decreases to $\sim 30 \mu \mathrm{S}$ as the magnitude of $E_{\perp}$ increases to $70 \mathrm{mV} / \mathrm{nm}$. Thus, in sample 1 , the conductance at the Dirac point exhibits a maximum at $E_{\perp}=0$, and monotonically decreases with perpendicular electric field of either polarity. Such behavior is exactly what one expects from tight binding calculations in the single particle picture [10], where a breaking of layer symmetry creates a gap in the band structure of BLG [10]. As the electric field that breaks this symmetry increases, the gap will also increase monotonically until it saturates at $\sim 300$ $\mathrm{meV}[10,12]$. This has been explicitly verified in optical studies [12]. In transport experiments [32-35] using BLG on $\mathrm{SiO}_{2}$ substrates, exponential decrease in conductance with $E_{\perp}$ has been observed, though disorder and charged impurities obscure the gap and give rise to variable range hopping. Thus, the behavior of sample 1 can be accounted for by $E_{\perp}$-induced band gap opening.

Sample 2, which has a mobility of $80,000 \mathrm{~cm}^{2} / \mathrm{Vs}$ and $V_{b g}=0.09 \mathrm{~V}$, displays a markedly different behavior. The color plot $G\left(E_{\perp}, n\right)$ and line traces $G(n)$ at fixed $E_{\perp}$ are shown in figures 2c-d. Interestingly, we observe a conductance minimum at $n=E_{\perp}=0$, which appears as the thin, short black line at the center of figure $2 \mathrm{c}$. As $E_{\perp}$ increase, $G$ increases to $\sim 100 \mu \mathrm{S}$. We note that the minimum conductivity at $n=E_{\perp}=0$ is as low as $0.1 \mu \mathrm{S}$, suggesting the presence of an intrinsic insulating state. Such an insulating state is not expected in the single particle picture, which evidently breaks down in this high mobility device with very little charged impurities. Recent transport studies $[17,19]$ have reported a similar non-monotonic dependence of the conductance minimum on $E_{\perp}$, and attributed this intriguing observation to an ordered ground state of bilayer graphene. Such states, according to various theoretical proposals, are: a gapped anomalous Hall state with broken time-reversal symmetry $[13,15,16,31,32]$, a gapped layer antiferromagnet state with broken time reversal and spin rotation symmetry [14-16], and a gapless nematic state which splits the $2 \pi$ Dirac point(s) into two $\pi$-Dirac points and breaks in-plane rotational symmetry $[37,38]$. The observations of Velasco et. al are perhaps best described by attributes of the gapped layer antiferromagnet state, though the exact nature of the ground state still remains controversial.

It is thus illuminating to compare behaviors and characteristics of these 2 samples. Single-particle physics is observed in sample 1; in contrast, sample 2, which has 4 times higher mobility and a CNP that is ten times closer to zero gate voltage, exhibits correlated electron behavior. Thus, high mobility and low charged impurities are crucial for the observation of many body physics.
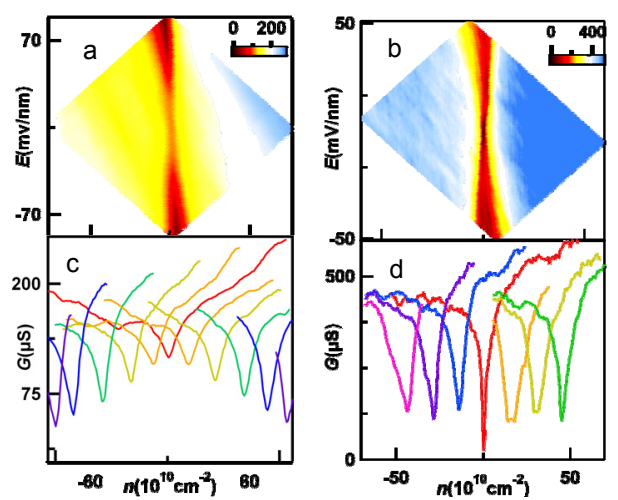

Fig. 3. Dual gate transport data at $B=0$ and $T=300 \mathrm{mK}$ for two different devices. a. $G(n, E)$ and line traces $G(n)$ at $E=85,71,57,42,28,14,0,-14,-28,-42,-57,-71,-85$ $\mathrm{mV} / \mathrm{nm}$, respectively (left to right). The line traces are laterally offset for clarity. b. $G(n, E)$ and line traces $G(n)$ at $E=-37.5,-25,-12.5,0, \quad 12.5,25$ and $37.5 \mathrm{mV} / \mathrm{nm}$, respectively (left to right). The line traces are laterally offset for clarity. 


\section{Transport Properties in the presence of a magnetic field}

In a large magnetic field, the charge carriers in BLG will adopt cyclotron orbits, which eventually coalesce to form landau levels (LLs). Within this picture BLG's energy spectrum is,

$$
E_{n}=(+/-) \frac{h e B}{m^{*}} \sqrt{N(N-1)},
$$

where $h$ is Planck's constant, $e$ is the charge of an electron and $B$ is the applied magnetic field. $N$ is an integer that denotes the index for each LL. This physical phenomenon manifest in transport measurements as quantized Hall conductivity,

$$
\sigma_{x y}=4 v e^{2} / h, \quad v=-3,-2,-1,1,2,3 \ldots
$$

Here $v=n h / B e$ is the LL filling factor denoting the electron density of the populated state.

From Eq. (5), the $N=0$ and $N=1$ orbital states are doubly degenerate, a degeneracy not found in MLG. Thus, the lowest LL of BLG is 8-fold degenerate. Such unusual symmetries have inspired significant theoretical and experimental efforts [18, 28, 40, 41, 42].

Here we explore this rich electronic structure of BLG in the presence of a magnetic field using our suspended dual gated structures. Figures $4 \mathrm{a}-\mathrm{d}$ are data taken from two different devices. In figure $4 \mathrm{a}$ the conductance (color) is shown as a function of $B$ and $n$. The colored bands that fan out from the Dirac point correspond to the quantum Hall plateaus, which move in the $n-B$ plane with slopes determined by their respective filling factors. Line traces at particular magnetic fields from the scan are plotted in figure $4 \mathrm{~b}$. When $B$ is increased from 0 , conductance plateaus emerge, in the order of $0,2,3,1$ $e^{2} / h$. These plateaus indicate that the 8 -fold degeneracy of the lowest LL is completely broken, in agreement with theoretical predictions [40] and previous experiments [18, $28,42]$. We note that a small plateau with value $1 / 2\left(e^{2} / h\right)$ is observed, which suggests the presence of a fractional state $[7,8,18]$, a prototypical example of electronic interactions in a $2 \mathrm{D}$ system.
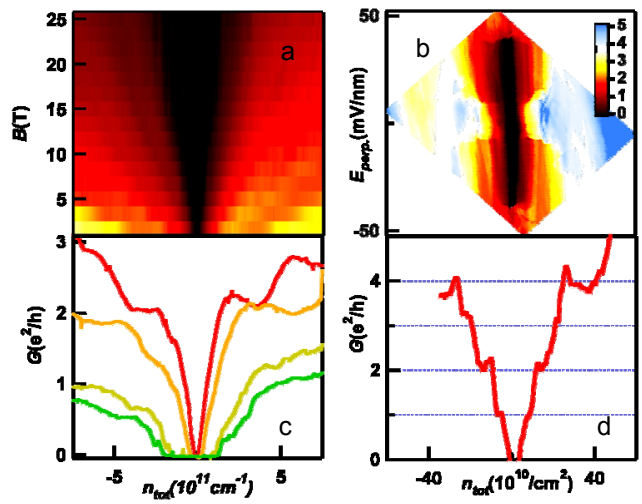

Fig. 4. Single and dual gate transport data in a magnetic field and $T=300 \mathrm{mK}$ for two different devices. a. $G(n, B)$ at $B=3.5 \mathrm{~T}$ and line traces $G(n)$ at $B=25,20,15,5$ T. b. $G(n, E)$ and line traces $G(n)$ and $E=-20 \mathrm{mV} / \mathrm{nm}$
We now examine the device in the presence of both $B$ and $E_{\perp}$. Figure $4 \mathrm{c}$ plots $G\left(n, E_{\perp}\right)$ at $B=3.5 \mathrm{~T}$. Here the $v=$ 0 plateau appears as the vertical black band centered at $v$ $=0$. It is surrounded by the red band, which corresponds to the $v=2$ plateau. Interestingly, the red band contains a "notch" near $E_{\perp}=0$, indicating the absence of the $v=2$ plateau therein. Thus, at $E_{\perp}=0$, only the $v=0$ and 4 plateaus are observed; at $E_{\perp}>15 \mathrm{mV} / \mathrm{nm}$, all degeneracies are broken, as shown in figure 4d [17].

To conclude this section we have found that using our suspended bilayer graphene samples of high quality we can probe the rich physics offered by the lowest LL of BLG. In our first high quality sample we modulated the carrier density and increased the magnetic field to a substantial 25T. This feat allowed us to observe a breaking of all the lower level degeneracies and at the highest field a fractional state. In our second high quality sample we tuned the $E$ and charge carrier density independently via our dual gates in a finite magnetic field. Varying the $E$ we were able to resolve all degeneracies including those more sensitive to layer polarization than spin or valley.

\section{Conclusion}

In this report we have discussed our transport studies of ultra-clean, suspended BLG with double gates. We described device fabrication in detail and the necessary post fabrication annealing for achieving high mobility samples. This regime of sample quality allows access to many intriguing physical phenomena. By comparing devices after current annealing with moderate and exceptional quality, we demonstrated distinct transport signatures of single particle physics and an interactioninduced correlated phase, respectively. Next, we explored quantum Hall effect physics of BLG in large magnetic fields. At our highest field values we observed evidence for a small fractional state, which is a hallmark of many body physics in two dimensional electron systems. At our lower fields we observed the breaking of lower LL degeneracies with a single and dual gate configuration. Taken together, our complete study underscores the versatility and richness of BLG, which has the capacity to host fundamentally intriguing physical phenomena with a possible avenue for real world electronics as well.

\section{Acknowledgement}

This work was supported in part by ONR/DMEA H94003-10-2-1003, NSF CAREER DMR/0748910, ONR N00014-09-1-0724, and the FENA Focus Center. D.S. acknowledge the support by NHMFL UCGP \#5068. Part of this work was performed at NHMFL that is supported by NSF/DMR-0654118, the State of Florida, and DOE.

\section{References}

1. K. S. Novoselov et al., Nature 438, 197-200 (2005).

2. K. S. Novoselov et al., Science 306, 666-669 (2004). 
3. M. I. Katsnelson, K.S. Novoselov and A.K. Geim, Nature Phys. 2 620-25 (2006).

4. A. F. Young and P. Kim Nature Phys. 5 222-6 (2009).

5. V. V. Cheianov, V. Fal'ko and B. L. Altshuler, Science 315 1252-5 (2007).

6. A. H. Castro Neto, F. Guinea, N. M. R Peres, K. S. Novoselov \& A. K. Geim, Rev. Mod. Phys. 81, 109 (2009).

7. X. Du, I. Skachko, F. Duerr, A. Luican, and E. Y. Andrei, Nature 462, 192 (2009).

8. K. I. Bolotin, F. Ghahari, M. D. Shulman, H. L. Stormer, and P Kim, Nature 462, 196 (2009).

9. S. Das Sarma, S. Adam, E. H. Hwang, \& E. Rossi, arXiv:1003.4731v2 (2010).

10. E. McCann, \& V. I. Fal'ko, Phys. Rev. Lett. 96, 086805 (2006).

11. K. S. Novoselov et al., Nature Phys. 2, 177 (2006).

12. Y. B. Zhang et al., Nature $459820-823$ (2009).

13. R. Nandkishore and L. Levitov, preprint, arXiv:1002.1966v1001 (2010).

14. O. Vafek, Phys. Rev. B 82, 205106 (2010)

15. F. Zhang, J. Jung, G. A. Fiete, Q. A. Niu \& A. H. MacDonald, Phys. Rev. Lett. 106, 156801 (2011).

16. J. Jung, F. Zhang, \& A. H. MacDonald, Phys. Rev. B 83, 115408 (2011).

17. R. T. Weitz, M. T. Allen, B.E. Feldman, J. Martin \& A. Yacoby, Science 330, 812-816 (2010).

18. W. Bao et al., Phys. Rev. Lett. 105, 246601 (2010).

19. J. Velasco Jr., et. al., arXiv:1108.1609

20. A.S. Mayorov et al., Science 333, 860-863 (2011).

21. G. Liu, J. Velasco Jr., W. Bao, and C.N. Lau, C. N. Appl. Phys. Lett. 92, 203103 (2008)

22. J. Velasco Jr., G. Liu, W. Bao, and C.N. Lau New J. Phys. 11, 095008 (2009)

23. J. Moser, A. Barreiro and A. Bachtold, Applied Physics Letters 91, 163513 (2007).

24. X. Du, A. Barker, I. Slachko and E. Y. Andrei, Nat. Nanotech. 3, 491 (2008)

25. K. I. Bolotin et al., Solid State Commun. 146, 351 (2008)

26. S. Das Sarma, E. H. Hwang and E. Rossi, Phys. Rev. B 81, 161407 (2010).

27. S. Xiao, J. H. Chen, S. Adam, E. D. Williams and M. Fuhrer, Phys. Rev. B 82, 041406 (R) (2010).

28. B.E. Feldman, J. Martin, and A. Yacoby, Nature Phys. 5, 889-893 (2009).

29. F. Miao, S. Wijeratne, Y. Zhang, U. Coskun, W. Bao and C.N. Lau, Science 317 1530-3, (2007)

30. S. Cho, \& M.S. Fuhrer, Nano Res. 4, 385 (2011).

31. W. J. Liang, M. Bockrath, D. Bozovic, J. H. Hafner, M. Tinkham and H. Park, Nature 411 665-9, (2001)

32. J. B. Oostinga, H. B. Heersche, X.L. Liu, A. F. Morpurgo, and L. M. K. Vandersypen, Nat. Mater. 7 151-7 (2008)

33. L. Jing, J. Velasco Jr., P. Kratz, G. Liu, W. Bao and C. N. Lau, Nano Lett. 10, 4000 (2010)

34. T. Taychatanapat, P. Jarillo-Herrero Phys. Rev. Lett. 105,166601 (2010)

35. K. Zou and J. Zhu, Phys. Rev. B. 82, 081407(R) (2010)
36. N. Nagaosa, J. Sinova, S. Onoda, A. H. MacDonald and P. N. Ong, Rev. Mod. Phys. 82, 1539 (2010).

37. F. Zhang, H. Min, M. Polini and A. H. MacDonald Phys. Rev. B 81, 041402 (R) (2010).

38. Y. Lemonik, I. L. Aleiner, C. Toke and V. I. Fal'ko, Phys. Rev. B 82, 201408 (2010).

39. O. Vafek and K. Phys. Rev. B 81, 041401 (2010).

40. Y. Barlas, R. Cote, K. Nomura and A. H. MacDonald, Phys. Rev. Lett. 101, 097601 (2008).

41. F. Guinea, A. H. Castro Neto and N. M. R Peres, Phys. Rev. B 73, 245426 (2006)

42. Y. Zhao, P. Cadden-Zimansky, Z. Jiang and P. Kim, Phys. Rev. Lett. 104, 066801 (2010). 